\title{
The abstraction of musical ideas
}

\author{
STEPHEN L. CHEW, LEAH S. LARKEY, SIGFRID D. SOLI, \\ JOE BLOUNT, and JAMES J. JENKINS \\ Center for Research in Human Learning, University of Minnesota, Minneapolis, Minnesota 55455
}

\begin{abstract}
Music presents information both sequentially, in the form of musical phrases, and simultaneously, in the form of chord structure. The ability to abstract musical structure presented sequentially and simultaneously was investigated using modified versions of the Bransford and Franks' (1971) paradigm. Listeners heard subsets of musical ideas. The abstraction hypothesis predicted (1) false recognition of novel instances of the abstracted musical idea, (2) confidence of "recognition" should increase as recognition items approximate the complete musical idea, (3) correct rejection of "noncases," which deviate from the acquired musical structure. Experiment 1 investigated sequential abstraction by using four-phrase folk melodies as musical ideas. Predictions 1 and 3 were confirmed, but the false recognition rate decreased as the number of phrases increased. Listeners were sensitive to improper combinations of phrases and to novel melodies different from melodies presented during acquisition. Experiment 2 investigated simultaneous abstraction using four-voice Bach chorales as musical ideas. Listeners spontaneously integrated choral subsets into holistic musical ideas. Musically trained listeners were better than naive listeners at identifying noncases.
\end{abstract}

There is more to music than individual notes, specific instruments, and specific parts. The complexity of music cannot be explained in terms of simple associations between notes. Instead, music has gestalt properties, in that the complexity of music arises from the patterns formed by the notes. Meyer (1956) has shown how the gestalt principles of pattern perception can be successfully applied to the perception of music. In a piece of music, the pattern of notes constitutes chords and phrases that may, in turn, form themes and movements within the piece. Such musical structure can be described abstractly. Music theorists have analyzed both the harmonic structure (e.g., Piston, 1962) and melodic phrasing (e.g., Benjamin, 1981) extensively. Several psychologists have also proffered schemes for the analysis of musical structure (Deutsch, 1980; Jones, 1978; Restle, 1970). In a recent review, Pick (1979) argues for a hierarchical organization of melodic structure. The relations between notes give rise to the structure of the music. To perceive a melody, the listener must abstract certain levels of structure from the relational properties of the notes.

This research was supported by grants to the Center for Research in Human Learning, University of Minnesota, from the National Science Foundation (NSF/BNS-77-22075) and the National Institute for Child Health and Human Development (T36-HD-07151 and HD-01136). We thank Joseph M. Waters for technical advice concerning the structure of baroque music, although we take complete responsibility for the content of this article. Leah S. Larkey is now at the University of California, Irvine. Sigfrid D. Soli is now at the University of Maryland. Joe Blount is now at the University of Minnesota, Morris. Requests for reprints should be sent to Stephen L. Chew, Center for Research in Human Learning, 75 E. River Road, Minneapolis, Minnesota 55455.
According to Pick (1979), listeners should be able to abstract the structure of music in much the same way as they abstract linguistic ideas or visual patterns. Bransford and Franks (1971) demonstrated that subjects spontaneously integrated the information contained in sentences into holistic, semantic ideas. They also showed that subjects abstract the prototype and transformations from a set of related geometric configurations (Franks \& Bransford, 1971). Music is a nonverbal, auditory code; it does not fit the usual dichotomy of visual or verbal codes. In music, information is transmitted both sequentially, as in sentences, and simultaneously, as in pictures. As with sentences, musical phrases are combined sequentially to form more complex musical themes. At the same time, music resembles visual patterns insofar as information is presented simultaneously through harmonic structure. We predicted that listeners would be able to abstract and integrate information presented through either type of musical structure.

We investigated the abstraction of musical ideas by adapting the paradigm used by Bransford and Franks (1971) in their study of the abstraction of linguistic ideas. This paradigm involves an acquisition phase and a recognition phase. In the present research, the holistic musical idea was broken down into component musical subsets, and a number of these subsets served as acquisition items in the acquisition phase. The holistic musical idea itself was not presented during acquisition. In the acquisition phase, the listeners performed an orienting task on each acquisition item. The orienting task served to focus the listeners' attention on a particular aspect of the musical stimuli and to constrain the types of strategies the listeners employed. Listeners were not instructed to memorize the acquisition items. After all the acquisition items had been presented, a surprise recognition 
test was given. The recognition items included (1) subsets of the holistic musical idea not presented during acquisition ("news"), including the holistic musical idea, (2) subsets previously presented during acquisition ("olds"), and (3) items that deviated in some manner from the holistic idea ("noncases"). Listeners judged the recognition items as either old or new and gave a confidence rating of their judgment.

The abstraction hypothesis makes three predictions. First, if listeners abstract and integrate the musical information presented during acquisition into holistic ideas, then listeners should have difficulty in discriminating olds from news during recognition. In other words, because specific items tend to be forgotten during the abstraction process, listeners should show false recognition of news, which, like the acquisition items, are valid subsets of the holistic musical idea. Second, the confidence of "recognition" should increase monotonically as the news approximate the holistic musical idea. If listeners are using the abstracted holistic idea to judge recognition items, then this fact should be reflected in the confidence ratings of the listeners. The holistic musical idea should be falsely recognized with the highest confidence. News that contain less information should also be falsely recognized, but with less confidence. Therefore, the confidence ratings should vary systematically with the amount of overlap of information shared by the new item and the holistic musical idea. Thus, both the amount and confidence of false recognition should be a positive function of how closely the news approximate the holistic idea. This pattern of results is taken as particularly striking evidence of the integration of component ideas into complete, complex ideas, because the complete idea, which is never presented in the acquisition set, is "recognized" with the highest confidence of all. Third, noncases should be correctly rejected. A noncase contains a deviation from the holistic idea and acts as a probe of the aspects of the musical structure that are remembered by the listener. A noncase that deviates from what is remembered by the listener should be treated differently from new items that conform to the abstracted musical ideas. By definition, abstraction refers to the apprehension of the information as structured in the holistic musical idea (e.g., news), not the random combination of components or the creation of a new structure (e.g., noncases). If the listener has abstracted the holistic idea, then the noncases should be perceived as novel.

Experiment 1 investigated the abstraction of melodic phrases, which are presented sequentially in music. Experiment 2 investigated the abstraction of harmonic structure, which is presented simultaneously in music.

\section{EXPERIMENT 1}

This experiment investigated the abstraction of melodic structure. An important kind of structure that may be abstracted from simple unaccompanied melodies is what Meyer (1973) calls conformant relationships, relationships in which "one (more or less) identifiable, discrete musical event is related to another such event by similarity" (p. 44). In simple folk melodies, such as those used in Experiment 1, many of the musical events or motives making up a given phrase appear again, although often transformed, in later phrases. This repetition with transformation is one of the properties that makes a piece of music a coherent entity rather than a sequence of random patterns. For example, one of the melodies used in Experiment 1 appears in Figure 1. The first measure of the second phrase is a transformation that simply lowers the pattern by a musical third, while maintaining the rhythm and contour. Other transformations may augment, simplify, or invert the sequence of notes. Other characteristic transformations appear throughout the piece.

Conformant relationships should provide listeners with the basis for abstracting the melodic structures that compose musical ideas. We examined this hypothesis by using a paradigm that has been successfully employed by Bransford and Franks (1971) to demonstrate the abstraction and integration of linguistic ideas. In the acquisition phase of the present experiment, listeners heard various subsets of component phrases from several folk songs, rather than sentences expressing portions of complex linguistic ideas. As with linguistic ideas, we expected that listeners would remember the abstracted musical ideas and not random conglomerations of phrases. Specifically, we expected that, during the subsequent recognition test, listeners would falsely recognize musical phrases that express the same con. formant relations as the musical ideas presented in the acquisition phase. We also anticipated that listeners would reject stimuli that deviate from the structure of the integrated musical idea. Finally, if musical and linguistic ideas are integrated in a similar fashion, we would
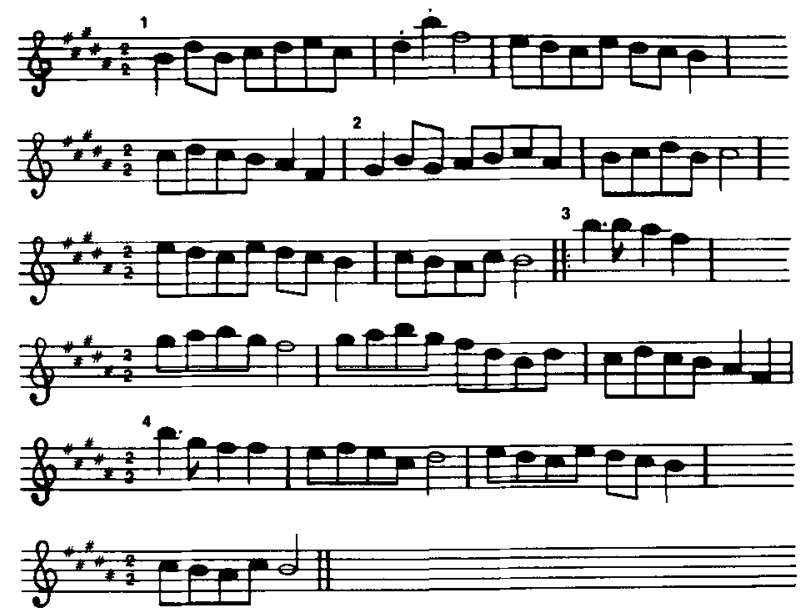

Figure 1. A melody with four component phrases used in Experiment 1. 
predict that the confidence of the listeners' recognition judgments should be primarily a function of the amount of melodic structure present in the stimuli. In other words, confidence ratings should be highest for the stimuli that exhaust all relevant relations of the musical ideas. These ratings should systematically decrease as the amount of structure present in a given stimulus also decreases. That is, confidence ratings should be greatest for renditions that contain all component melodies of the musical idea and decrease as the number of melodic phrases present in a given rendition decreases.

\section{Method}

Subjects. The subjects were 16 undergraduate student volunteers at the University of Minnesota who received class credit for their participation in the experiment. The musical backgrounds of these subjects varled widely. Three were music majors, 2 had no formal musical training, and the remaining 11 had a mean of 5.4 years of group or individual instrumental training received during their primary or secondary education.

Stimuli. The experimental stimuli were made from melodies chosen from various collections of Western European folk music. This source was used to ensure that, while specific melodies were unlikely to be familiar, their melodic and harmonic structures would be fairly common and well known. Only 16-bar melodies written in major keys were selected. The 16-bar pattern had to be composed of four 4-bar phrases that did not repeat, with the final phrase ending unambiguously on the tonic note of the melody's key. Except for the presence of repeated phrases, most of the surveyed melodies conformed quite closely to these constraints. The six melodies selected were: "Annchen von Tharau," "Twin Sisters," "Folk Dance," "Making Hay," "Meg," and "Sah' ein Knab', ein Roslein Stehn." These melodies will subsequently be designated by the letters $\mathrm{A}$ through $\mathrm{F}$.

Melodies that were not originally in $\mathrm{C}$ major were transposed into that key so that key signature could not be used to distinguish the melodies. Melodic lines were performed unaccompanied, without their original harmony or chords, by a professional pianist, for tape recording with high-quality audio equipment. The pianist was instructed to perform each melody in the appropriate musical style, as designated on the score, and to insert a very brief silent interval between phrases for tapesplicing purposes.

The stimuli for the acquisition task and the old and new stimuli for the recognition task were made from Melodies A, B, and C. One-, two-, three-, and four-phrase melodic segments, analogous to the linguistic stimuli with varying amounts of a complex semantic idea (Bransford \& Franks, 1971), were spliced from multiple copies of the original recordings. Four onephrase stimuli ("ones") were made from each melody by excising single four-bar phrases. Three two-phrase stimuli ("twos") and two three-phrase stimuli ("threes") were also made by removing the appropriate number of adjacent phrases in their original order from each melody. "Fours," of course, consisted of the entire, intact melodies. A total of 10 stimuli were made from each melody in this fashion. Half of these stimuli from each melody together formed the 15-stimulus set for the acquisition task, with 5 stimuli from this set appearing in the recognition task as the old stimuli. All four phrases occurred somewhere in the acquisition set. The remaining 15 stimuli from Melodies A, B, and C composed the new stimuli in the recognition task. In addition, five melodic phrases designated as noncase stimuli were formed by splicing together phrases from different melodies that were presented as temporally adjacent stimuli in the acquisition task. These stimuli were either two or three phrases in length. Finally, five "different" stimuli, two ones, two twos, and one three, were made from Melodies D, E, and F, which were not presented during acquisition. Thus, to sum- marize, the acquisition task consisted of 15 stimuli, 5 each from Melodies A, B, and C. The 30 stimuli for the recognition task consisted of five of the acquisition stimuli (the olds), the 15 stimuli from Melodies A, B, and C that had not been presented during acquisition (the news), 5 noncases, and 5 different stimuli.

The stimulus recording for the acquisition task was made so that no stimuli from the same melody occurred on adjacent trials, with $5 \mathrm{sec}$ of silence separating each trial. In addition, no fours were presented in the acquisition task. Four recordings containing the 30 recognition stimuli were also prepared. These tapes differed only in stimulus order for counterbalancing purposes. Recognition stimuli were also separated by 5 -sec silent intervals.

Procedure. The listeners were divided into four groups for the purpose of counterbalancing the presentation order in the recognition task. Listeners in each group were told that the experiment consisted of three parts that would be explained separately.

Acquisition. The listeners were told that their task was to rate segments of melodies on three dimensions derived from the semantic differential (Osgood, Suci, \& Tannenbaum, 1957): peaceful-lively, unpleasant-pleasant, and weak-powerful. Two brief segments from familiar melodies were played first to familiarize the listeners with the task. The recording of the 15 acquisition stimuli was then played three times so that listeners could rate one dimension separately each time through the recording. The rating task was used to ensure that listeners attended to the overall quality of the melody rather than specific notes. The acquisition stimuli contained two ones, two twos, and one three from each of Melodies A, B, and C. During acquisition, listeners were not informed that they would receive a memory test on these stimuli.

Recognition. Following acquisition, the listeners were given a brief break. They were then told that they would hear 30 melodic segments in a memory test. They were asked to respond "yes" or "no" to each segment, indicating whether they had heard that exact segment during acquisition. They were also asked to indicate how confident they were in their judgment by making a confidence rating on a 5-point scale, with " 1 " indicating very low confidence and " 5 " indicating very high confidence. The 30 stimuli in the recognition task included 5 old, 15 new, 5 noncase, and 5 different stimuli, as described above. A single recognition test was given to each group of listeners. Group 1 received the randomly ordered stimuli in an order of presentation designated 1-30. Group 2 received the stimuli in the order 16-30 followed by 1-15; Group 3 received the order 30-1; and Group 4 received the order 15-1 followed by 30-16. Following the recognition test, the listeners were asked to describe their musical background and training. They were also asked to indicate how many melodies they thought they had heard on the acquisition tape and whether any of the melodies were familiar.

\section{Results}

Ratings. Ratings on the semantic differential were transformed to normal scores as follows: The mean and standard deviation were computed from each listener's ratings on each dimension. Ratings were then expressed in standard deviation units with the mean as zero. Thus each of the 15 acquisition melodies received a pooled rating, which located the melody in the three-dimensional space defined by the three rating dimensions. Figures 2 and 3 show the locations of the 15 melodies in the space, two dimensions at a time. The three distinct clusters of points in the space show that the acquisition stimuli that came from the same melody received similar ratings. 


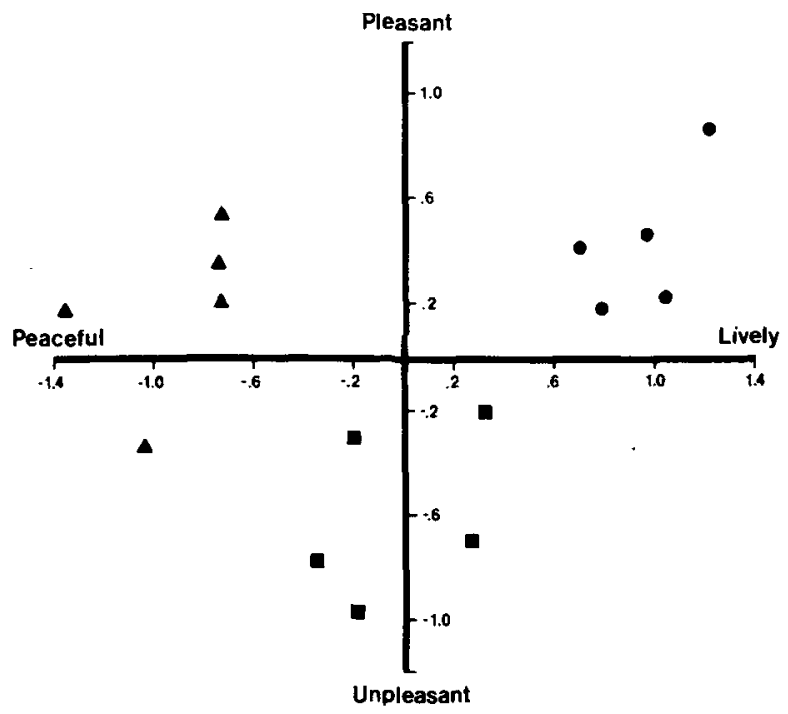

Figure 2. A semantic differential plot for derivations from three folk melodies. The dimensions of activity and affect are represented.

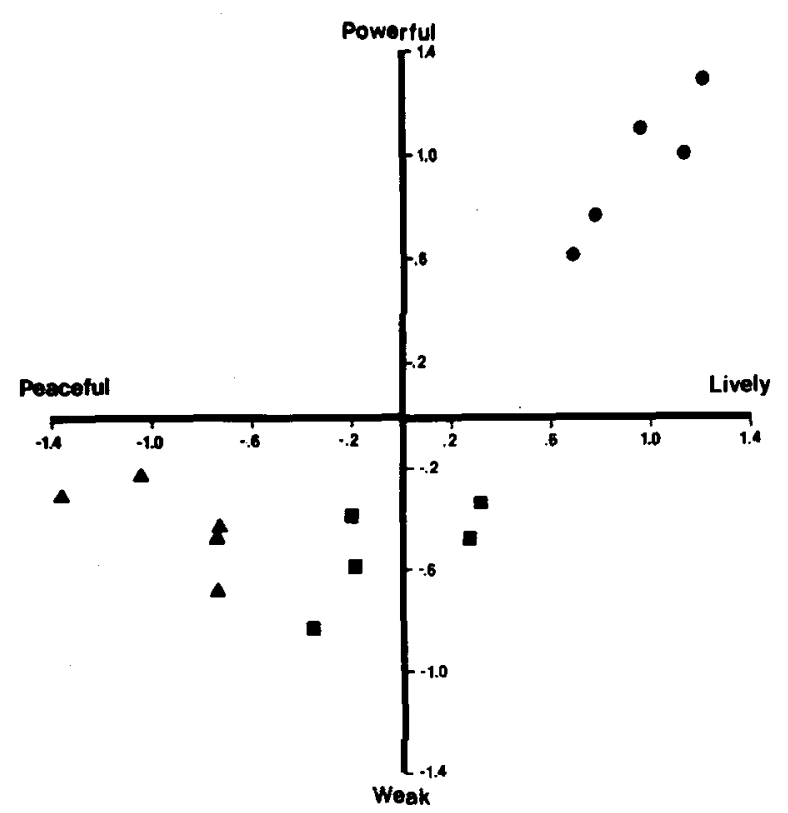

Figure 3. A semantic differential plot for derivations from three folk melodies. The dimensions of activity and potency are represented.

Recognition. Listeners' responses were given numerical scores by assigning a positive value to the confidence ratings of the "yes" responses, which indicated either correct recognition of an old item or false recognition of a new item, and a negative value to the confidence ratings of the "no" responses. This yields a 10-point rating scale from -5 to +5 , excluding 0 . Old, new, noncase, and different items of the four different lengths were examined separately.

Figure 4 shows the mean confidence ratings for the items in all categories. Positive scores for olds represent correct recognition. The scores for the olds composed of one, two, and three phrases were $1.72,2.63$, and 2.06 , respectively, indicating that listeners were moderately confident that they had heard these stimuli in acquisition. Positive scores for the news represent false recognition. Mean ratings for news with one, two, three, and four phrases were $1.10,1.17, .06$, and .06 , respectively, indicating only a small amount of false recognition. Olds received significantly higher scores than news when ones, twos, and threes from the same melodies were compared simultaneously $[\mathrm{t}(79)=3.13$, $\mathrm{p}<.001]$. When the ones, twos, and threes were analyzed separately, only the twos showed significantly higher scores for olds than for news $[t(31)=2.28, p<.05]$. The news, then, were neither falsely recognized nor correctly rejected. It is interesting to note that listeners could have rejected complete melodies (fours) unequivocally if they had noted that the acquisition set included

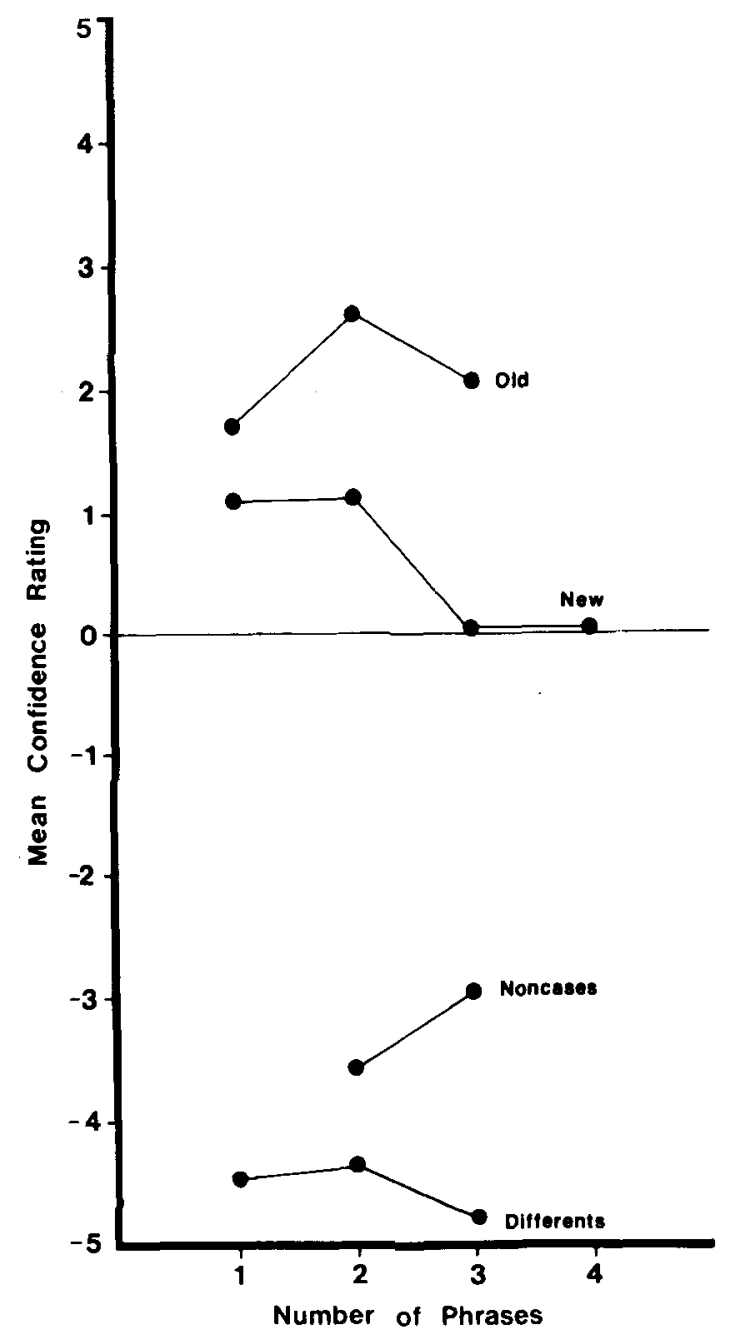

Figure 4. Mean confidence ratings for olds, news, noncases, and differents. 
no complete melodies, but they did not. If they had rejected complete melodies on this basis, we would have found more confident rejection of four-phrase new melodies than three-phrase (and other) new melodies. Instead, both threes and fours received a score of .06 .

The scores for the noncase melodies composed of two and three phrases were -3.54 and -3.06 , respectively. The scores for different melodies of one, two, and three phrases were $-4.47,-4.38$, and -4.81 , respectively. These data indicate robust correct rejection of melodies that were not heard before. Totally new melodies (differents) were more confidently rejected by listeners than were new melodies composed of phrases from old melodies (noncases) $[\mathrm{t}(31)=8.17$, $\mathrm{p}<.001]$.

\section{Discussion}

Several differences are apparent between these results and those found for integration of linguistic ideas (Bransford \& Franks, 1971). Bransford and Franks found no evidence for specific memory. Subjects treated news exactly as if they had heard them before. In contrast, the listeners in the present experiment showed specific memory. They were more confident in their recognition of olds than they were in their (false) recognition of news.

A second important difference between the results of the present experiment and the results of Bransford and Franks (1971) is that those investigators found a monotonic increase in confidence as similarity to the complex idea increased. This monotonic ordering was not evident in the present experiment. In fact, the complete musical ideas (fours) were tied for the lowest confidence scores among the new stimuli. Perhaps length was too salient in our acquisition set, which had more one-phrase and two-phrase stimuli than three-phrase stimuli. This possibility is supported by the fact that Flagg (1976), using sentences, found similar recognition results for new items when he presented only ones during the acquisition phase.

The results described above provide only equivocal evidence for the abstraction of musical ideas. There are at least two possible interpretations of these results. First, listeners may not have abstracted the complete melodies but may have learned only the stimuli in the acquisition set. If this were strictly true, new melodies should have been correctly rejected just as the noncases were, because both noncase melodies and news contain parts of what listeners had heard before. Certainly, the fours should have been rejected, since they were longer than any of the acquisition melodies. The fact that news were not correctly rejected argues against this explanation. A second possibility is that the result may have been due to stimulus and task variables. The acquisition set was fairly short, and listeners heard the set three times during the rating task. These conditions would facilitate specific memory. In addition, the large number of ones and twos in the acquisition set may have prevented the longer recognition melodies from being falsely recognized. The coherence of phrases of a melody may also be fundamentally different from that of linguistic ideas. Complex linguistic ideas can be broken down into syntactically acceptable sentences that embody some subset of meaning of the whole idea. However, it is not clear that subsets of component phrases may adequately reflect the complex musical idea. In other words, there may be some property of the whole melody, an abstract coherence or identity among the phrases, that is lost when only subsets of the component phrases are presented. If this is the case, then the subsets of phrases of a melody would each form a different, but related, musical melody. We would then expect that listeners would not be able to abstract the subsets of phrases into the complete melodic idea. Still, the fact that related acquisition items overlapped in phrases and that phrases shared some properties in common would allow listeners to apprehend some qualities of the overall melody. This would explain the correct rejection of noncases and differents. The ratings on the semantic differential scale support this idea (see Figures 2 and 3 ). The ratings show that stimuli from the same melody received similar ratings, but that the ratings for each melody were different. These ratings indicate that each melody may have suggested a slightly different "mood" to the listeners. If this were the case, listeners could have responded to the change in mood in the middle of a noncase melody on that basis.

A second experiment was conducted to eliminate the possible sources of the differences between the results described above and those of Bransford and Franks (1971). The abstraction of musical ideas may be fundamentally different from the abstraction of linguistic ideas, perhaps because linguistic ideas have referential meaning and musical "ideas" generally do not. On the other hand, the integration of linguistic ideas may be similar to many kinds of abstraction, including that of musical ideas. In Experiment 2, we investigated the abstraction of musical ideas whose four components occur simultaneously instead of sequentially. This eliminated the salience of length and maintained the integrity of the melodic line, which may have prevented abstraction from occurring in Experiment 1. Furthermore, we controlled for task variables by using a larger acquisition set and presenting it only once.

\section{EXPERIMENT $2^{1}$}

Pratt, Rushford, and Jarrett (Note 1) used the paradigm of Bransford and Franks (1971) to study the abstraction of musical ideas presented simultaneously. Using Bach chorales for musical ideas, ${ }^{2}$ they found only a small trend to suggest that abstraction of the musical ideas had occurred. However, their study had 
serious methodological problems that may have been responsible for the equivocal results.

In Experiment 2, we modified the method of Pratt et al. (Note 1) in several ways. The major change was the use of an orienting task during the acquisition phase of the experiment. Pratt et al. allowed the listeners to decide what strategies, if any, they would use to learn the chorales. These instructions would be particularly vague to musically naive listeners, who had no previous experience with baroque chorales. In the present study, a simple orienting task was used for all listeners. Listeners heard a chorale and then a number. The listeners' task was to count two numbers backward from the given number and then silently hum the chorale and tap out the rhythm. This task focused attention on the chorales, constrained the strategies that the listeners could employ, and provided some feedback about the effectiveness of the strategy. Another difference was that the listeners heard each instrument play a scale before the acquisition phase began. This familiarized the listeners with the instruments and provided practice at the orienting task.

Bach chorales are particularly well suited for studying the abstraction of musical ideas that are presented simultaneously. The music of Bach is the quintessence of the contrapuntal harmonic technique of the baroque era. Baroque music is highly structured and has been analyzed extensively (e.g., Piston, 1962). Bach's chord choice, chord movement, part writing, and hence, his chorale harmonization typify the baroque style. The chorale harmony does not merely support the main melody line, but each part in these chorales is a separate melody. The main melody of the chorale is presented in the soprano line; to this melody are added three new and different melody lines to enhance and expand the theme of the chorale. Since each voice follows the baroque conventions of good melody writing, each voice can stand alone as a melody. At the same time, the harmony among the voices of the chorale also follows the baroque conventions of chord choice and chord movement. Thus, each voice acts both as a melody in and of itself and as a harmony for the other voices.

We predicted that listeners would be able to abstract the complex chord structure of chorales. Since the chord structure arises from the interrelations between the lines of a chorale, component subsets of the lines of a chorale would therefore yield constituent subsets of the chord structure. Listeners should spontaneously abstract the chord structure from these subsets of voices and integrate this information into the complex chord structure present in the whole chorale. This should result in false recognition of new chorale subsets that contain the same chord structure as the whole chorale ("news"). Furthermore, the amount and confidence of (false) recognition should increase as the chord structure of new chorales more closely approximates the chord structure of the whole chorale. Spe- cifically, the abstraction hypothesis predicts that, in the recognition task, listeners' confidence ratings of news should increase monotonically with the number of voices present in a new chorale subset. It further predicts that noncases, which deviate from the abstracted harmonic structure, should be correctly rejected.

Music also allows the role of expert knowledge in idea acquisition to be studied. Bransford and Franks (1971) used sentences that expressed four component concepts as linguistic ideas. The level of linguistic expertise of listeners was consistently high. Thus, Bransford and Franks did not vary the level of expertise of their listeners. While most people are linguistically proficient, the range of musical expertise in the population is quite varied. Thus, the level of musical experience can be controlled.

In addition to the monotonic prediction, we also predicted that musically trained listeners would show greater abstraction of musical structure than musically naive listeners. Pick (1979) asserts that musicians are able to perceive more complex levels of melodic structure. A study by Dowling (1978b) supports this idea. Musical experts perceived both the contour and relative interval sizes of novel melodies, whereas untrained listeners perceived only the contour.

Finally, musically trained listeners should retain more specific information than naive listeners. In addition to perceiving more complex levels of melodic structure, musicians are better at identifying specific lines and specific instruments (e.g., Dowling, 1978a). Therefore, the musically trained listeners should be more adept at remembering which subsets they had heard during the acquisition phase.

\section{Method}

Subjects. The naive listeners were 15 students enrolled in introductory psychology classes at the University of Minnesota. They received extra credit for their participation. These listeners had not had any formal musical training. The musically trained listeners were eight music majors. Seven of these were enrolled in their 2nd year of ear training classes at the University of Minnesota. The eighth listener had already completed the 2-year ear training sequence. Their average amount of formal musical training was 9.25 years, with a standard deviation of 3.03. They were paid for their participation.

Materials. The stimuli were generated from excerpts of four Bach chorales. These excerpts were matched for length, and all four were in the key of $F$ major. The Bach chorales used were "Das walt' Gott Vater und Gott Sohn" (BWV 290), "Herr Jesu Christ, wahr's Mensch und Gott" (BWV 127), "O Ewigkeit, du Donnerwort" (BWV 20), "O Welt, ich muss dich lassen" (BWV 244, BGA 4, 164). Each chorale consisted of four lines or voices. From these four-voice chorales, one-voice, two-voice, and three-voice subsets were formed. A three-voice subset ("three") was derived from the four-voice Bach chorale by removing a particular line. For example, a three-voice sopranotenor-bass subset would be formed by removing the alto line. Two-voice subsets ("twos") were formed by leaving out two lines, and one-voice subsets ("ones") were derived by leaving out three lines. The "fours," of course, were the complete Bach chorales. Thus, 15 renditions could be derived from one Bach chorale. 
Six noncases were formed by using three lines from one chorale and one line from a different chorale. The deviations that resulted from combining lines of different chorales were striking departures from the baroque conventions of chord structure and chord movement. However, the three regular voices from one chorale tended to mask any dissonances caused by the deviant voice. Hence, noncases were four-voice chorales that contained noticeable, but not necessarily conspicuous, deviations from the structural relations of Bach chorales.

A quartet consisting of oboe, clarinet, French horn, and bassoon performed the chorales. The oboe played the soprano line, the clarinet played the alto line, and the French horn and bassoon played the tenor and bass lines, respectively. The 4 fours were recorded, as well as 16 ones, 16 twos, 14 threes, and 6 noncases. Six renditions, two ones, two twos, and two threes were chosen to be old items and were recorded twice. One recording was used for the acquisition phase, and the other recording was presented during the recognition phase. A recording of each instrument playing 1-octave scale was also made.

The acquisition list consisted of 24 chorale subsets, six derivations from each complete Bach chorale. Eight ones, eight twos, and eight threes were presented during acquisition, but no fours were presented. The renditions used in the acquisition phase were chosen such that all four lines of each Bach chorale were presented. The acquisition items were randomly ordered for presentation, with the constraint that no two derivations from the same Bach chorale occur consecutively.

The recognition list included 24 chorale subsets not presented during acquisition (news), 6 chorale subsets that had been presented during acquisition (olds), and the six noncases. The new items consisted of four fours, eight ones, eight twos, and four threes. Old items were different performances of chorale subsets that had been presented during acquisition. The six olds consisted of two ones, two twos, and two threes. Recognition items were randomly ordered, with the constraint that no two derivations from the same Bach chorale occur consecutively.

Procedure. Listeners were tested in small groups of one to four people. Stimulus materials were presented over a loudspeaker from a high-fidelity reel-to-reel tape recorder.

Acquisition. The following orienting task was used during acquisition. Listeners heard a rendition, and then the experimenter said a number. Listeners wrote down that number and the two next smaller numbers. Then they hummed the melody silently to themselves while tapping out the rhythm with their foot. Pilot studies had shown that naive listeners had no difficulty performing this task. Thus listeners were forced to attend to the stimuli well enough to retain them over the delay and perform the humming task. Listeners were not instructed to learn the chorales, nor were they informed about the recognition task.

Listeners were given four practice trials on the four 1-octave scales. This familiarized them with the orienting task and with the sound of the instruments.

Recognition. After all 24 acquisition items had been played, a surprise recognition test was given. There was a delay of approximately $2 \mathrm{~min}$ while answer sheets were distributed, instructions were given, and the recognition tape was readied. Listeners judged the 36 recognition chorales as "old" or "new" and rated the confidence of their judgment on a 1-5 scale. A 1 indicated a guess, and a 5 meant "very sure."

After all the recognition items had been presented, the listeners were asked to describe their musical training and background.

\section{Results}

Listeners' responses were transformed into numerical scores as in Experiment 1 by assigning a positive value to the confidence rating of "old" responses and a negative value to the confidence rating of "new" responses. This yields a 10-point rating scale from -5 to +5 , excluding 0 . Olds, news, and noncases were examined separately.

First, an overall average was calculated for each number of voices. The mean confidence ratings for the news as a function of the number of voices are presented in Figure 5. Since these are all news, a negative rating is a correct rejection and a positive rating indicates a false recognition. Both trained and naive listeners give monotonically increasing functions. Although ones showed overall correct rejection, the rate of false recognition of chorale subsets increased as the number of voices in a subset increased. In other words, as the number of voices in a new item increased, it was more likely to be falsely recognized by listeners. Furthermore, listeners became more confident of their (false) recognition as the number of voices in a subset increased. Trained listeners tended to reject more news than did naive listeners, except for fours. Mean ratings for trained

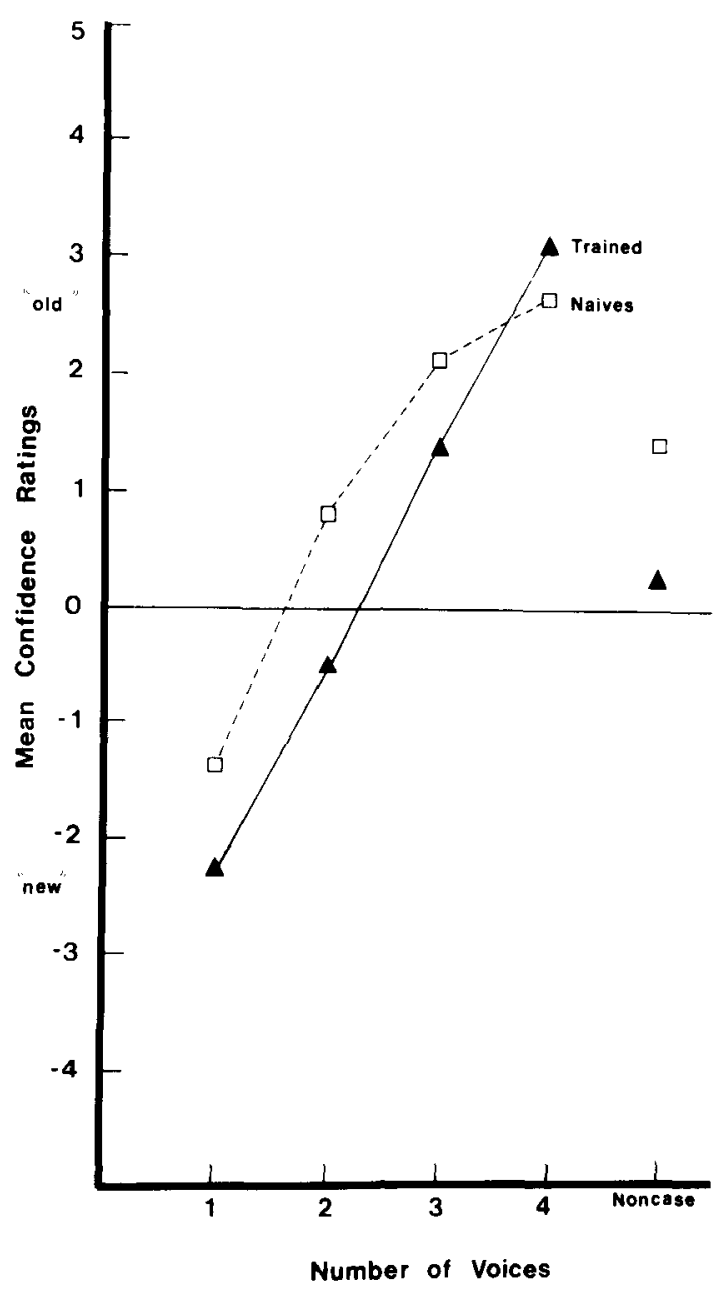

Figure 5. Mean confidence ratings of musically trained and naive listeners for news and noncases. 
listeners were $-2.33,-.5,1.38$, and 3.16 for ones, twos, threes, and fours, respectively. Mean ratings for naive listeners were $-1.38, .81,2.12$, and 2.68 for ones, twos, threes, and fours, respectively.

Standard statistical tests are not applicable to these results. Many of the renditions are interdependent. Interdependence exists since renditions derived from the same Bach chorale often had voices in common. This lack of independence would cast serious doubt on the validity of a probability statement derived from a parametric statistical test.

Ordinal comparisons of recognition ratings were carried out for each chorale. Rendition $\mathrm{X}$ was predicted to have a greater confidence rating than Rendition $Y$ if $X$ contained all the voices of $Y$ plus any other voices. So, for example, an oboe-clarinet-bassoon rendition should have a greater confidence rating than the clarinetbassoon rendition derived from it, and both of these chorales should have greater confidence ratings than the one-part oboe rendition. Thirty-four predictions can be derived from this hierarchical analysis of chorale renditions. Naive listeners confirmed 29 predictions, and all 34 predictions were confirmed by experts. In order to test the statistical significance of these results, a Monte Carlo procedure was run 10,000 times to derive the probability distribution of the number of predictions confirmed by chance (cf. Bransford \& Franks, 1971). From this distribution, it was determined that the probability of the number of predictions confirmed by listeners was due to chance was significantly small for both trained $(p<.001)$ and naive $(p<.001)$ listeners.

A post hoc examination of the data revealed that this monotonic relationship could not be accounted for by the predominance of a particular chorale or a particular voice for either naive or trained listeners. The data for each chorale were examined separately, and the monotonically increasing trend was found in each instance. The judgment of a rendition as "old" or "new" was not systematically related to the presence or absence of a particular voice but, rather, was related to how many voices the rendition contained.

The average ratings of noncases is also shown in Figure 5. The mean ratings of noncases were .23 for trained and 1.48 for naive listeners. Noncases were novel chorales composed of four lines: three lines from one chorale and one line from a different chorale. Therefore, the relevant comparison is with the fours (i.e., the complete Bach chorales), since noncases also had four voices. Neither naive $[t(14)=2.64, p<.05]$ nor trained $[\mathrm{t}(7)=5.28, \mathrm{p}<.01]$ listeners treated noncases the same as fours, even though both were novel and had the same number of voices. This effect was particularly striking for trained listeners (see Figure 5). A $t$ test was performed on the difference between fours and noncases for trained listeners vs. the difference between fours and noncases for naive listeners. It was found that trained listeners were significantly better at discriminating noncases from fours than were naive listeners $[\mathrm{t}(21)=2.36, \mathrm{p}<.05]$.

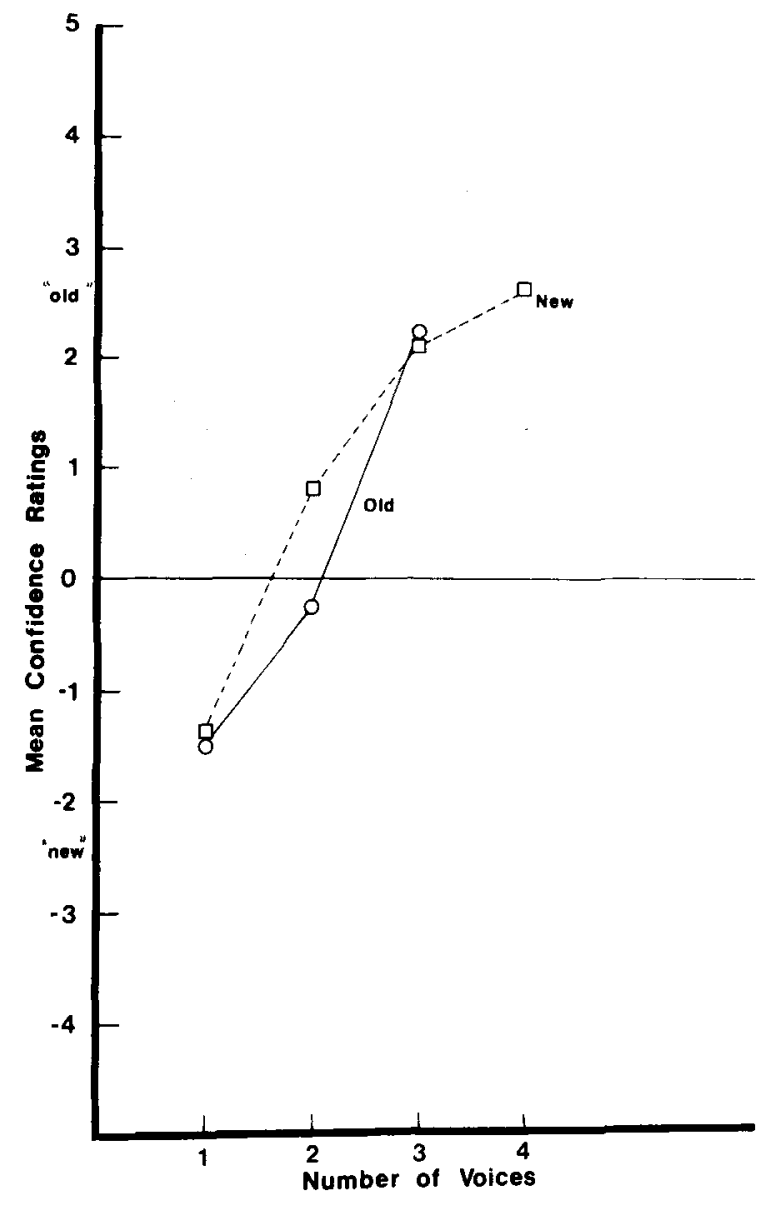

Figure 6. Mean confidence ratings of naive listeners for olds vs. news.

Although there were not enough old items in the recognition test to provide a fair test of specific memory for chorale subsets, the results are suggestive. The mean ratings of naive listeners for olds and news are plotted as a function of the number of voices in a chorale subset in Figure 6. For olds, a positive value indicates overall correct recognition and a negative value indicates overall incorrect rejection. No fours were olds, since these were presented only during recognition. The mean ratings of olds for naive listeners were -1.5 , -.27 , and 2.27 for ones, twos, and threes, respectively. Old items were recognized in essentially the same way as new items. Musically naive listeners showed no evidence for specific memory. The results for trained listeners suggest that they may be able to distinguish between olds and news (see Figure 7). While the differences were not significant, olds were rated consistently higher than news. The mean ratings of olds for trained listeners were $-1.44,-.19$, and 3.44 , respectively.

\section{Discussion}

The second experiment presents clear evidence for the abstraction of the simultaneous component of musical structure. Listeners spontaneously abstracted the structural relations from the ones, twos, and threes 


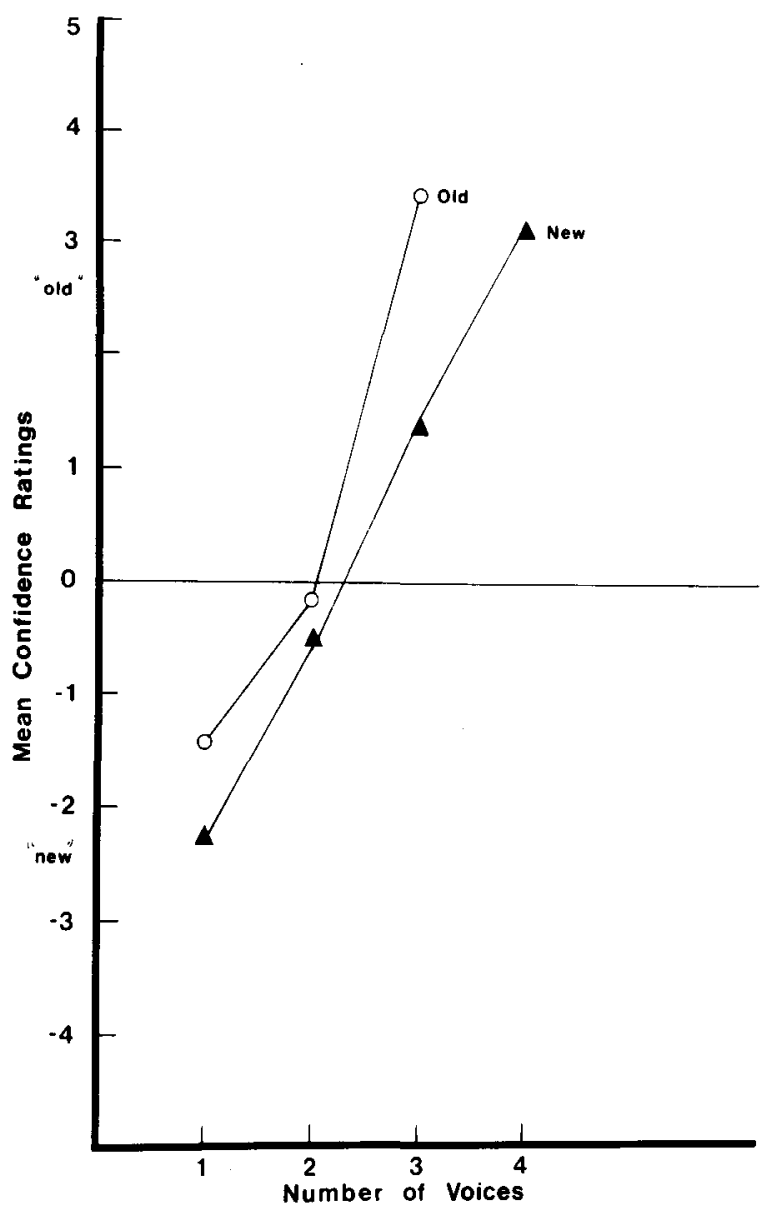

Figure 7. Mean confidence ratings of musically trained listeners for olds vs. news.

presented during acquisition and integrated these relations into the complete Bach chorales. This occurred despite the fact that no two acquisition items derived from the same Bach chorale were presented consecutively during acquisition. Listeners falsely recognized many news that expressed the same structural relations as the acquired ideas. Furthermore, the confidence of (false) recognition of news increased as their chord structure increased in similarity to the abstracted ideas. That is, listeners' confidence that the new items were old (i.e., false recognition) was greatest for fours and decreased systematically for threes, twos, and ones. This occurred despite the fact that no four-voice chorales had been presented in acquisition. Finally, listeners were able to discriminate noncases from fours. This indicates that listeners acquired four integral ideas and were not merely responding to the number of voices present in a new item. In other words, this rules out the possibility that listeners were adopting a guessing strategy based on the number of voices in a given recognition item.

The results of Experiment 2 support the gestalt notion that listeners perceive the relations among the notes. Specific instruments and voice combinations were quickly forgotten in favor of remembering holistic ideas. These findings are consistent with Pick's (1979) hierarchical analysis of musical structure. Listeners were able to perceive the musical structure as a function of the extent of their musical expertise. Musically trained listeners were significantly better at detecting noncases than were musically naive listeners. This suggests an overall superiority for musically trained listeners. They were better at both abstracting melodic structure and remembering specific information, in this case the instrument or voice combination.

Both trained and naive listeners showed reliable discrimination of noncases. Unlike the results of Experiment 1 , however, neither group showed correct rejection of noncases. There are several possible reasons for this outcome. The musical expertise of the listener obviously played a role. Even though trained listeners have a high degree of musical expertise relative to the naive listener, their level of competence with music in general and baroque music in particular cannot compare to an average person's linguistic expertise. By using sentences as linguistic ideas, Bransford and Franks (1971) allowed listeners to utilize their highly proficient skill of sentence comprehension. Our results suggest that the correct rejection of noncases in their studies was due at least in part to the high level of linguistic expertise of the listeners. In the present experiment, the level of musical expertise, while affecting the detection of noncases, may not have been sufficiently high to result in correct rejection of noncases.

Another possible reason why noncases were not rejected is that the structural information was presented simultaneously to the perceiver. Chord structure is similar to a picture, in that all information is presented at the same time. Franks and Bransford (1971) used visual patterns as stimuli and found that viewers abstract a prototypical visual pattern and the transformations by which the patterns were derived. They also showed that not all violations of the valid transformations of the prototype were equally salient. That is, noncases differed in how easy they were to detect. This supports the idea that when information is presented coinstantaneously, the salience of the noncases will vary, depending on the type of structural deviations contained in the noncase. In the present experiment, the noncases were constructed in a conservative manner, in that the structural deviations were subtle. First, the deviant line was masked by the other three chorale lines. Chew, Blount, and Waters (Note 2) found that two-voice noncases, composed of one line from one chorale and one line from a different chorale, were more noticeable to listeners than four-voice noncases. This indicates that the deviant musical structure was masked in the four-voice noncases. Second, noncases represented deviations of the baroque conventions, but the structure of noncases would be acceptable examples of more modern musical 
styles. The most salient cue for rejecting noncases is that, unlike the acquisition items, noncases do not have a baroque musical structure. This requires that the listeners be able to recognize the baroque musical structure of the acquisition items. Finally, unlike a picture, music also has a sequential aspect, that is, the melody, which limits the time for the listener to perceive the chord structure. The chord structure is not static like a picture, rather it changes with the melody. That is, the melody constrains the time available to the listener to perceive a chord before it is replaced by a different chord.

In short, no serial cue existed with which to identify noncases. The constituent voices of both noncases and fours had been presented during acquisition; only the relationship between the voices, that is, the chord structure, differed between the two. The chord structure of fours was the same as that presented during acquisition, but the pattern of interrelations among the voices of noncases was completely novel. Thus, the noncases were the proper controls to test for the abstraction of musical structure. The reliable discrimination of noncases from fours, then, indicates that the chord structure of the chorales was what was remembered by the listeners.

\section{GENERAL DISCUSSION}

As an auditory, nonverbal code, music provides some interesting insights into human cognition. The results of Experiments 1 and 2 demonstrate that all listeners have an awareness of certain global properties of music. In Experiment 1, the performance on noncases indicates that listeners had a strong sense of the coherence of melodic phrases, that is, which melodic phrases fit together to form complete melodies. In addition, different items were confidently rejected. These two results indicate that listeners had a strong sense of the global identity of the musical ideas. In Experiment 2, the abstraction of chord structure required the perception of at least two aspects of the global harmonic context. First, listeners had to be aware of the global simultaneity of the four voices. In other words, although listeners never heard more than three voices together during acquisition, they were aware that all four voices occurred simultaneously in the full chorale. Serafine (Note 3) has referred to this as "thematic synthesis and abstraction." In addition, listeners were able to create the timbral blend of the four instruments even though no more than three instruments were presented at any time during acquisition. Serafine refers to this as "timbral synthesis and abstraction." These two abstraction processes must be operating for the abstraction of chord structure to occur.

The fact that listeners spontaneously integrate ideas presented simultaneously is evidence for the generality of the abstraction process. Abstraction of information is obviously not limited only to linguistic and visual stimuli. In addition, Experiment 2 demon- strated that the ability to abstract information is related to the level of expertise. Experiment 1 showed that not all information is structured in such a way that permits abstraction, and yet Experiment 2 supports the idea that there is a general ability to abstract and integrate information given suitable structure and context.

The present experimental paradigm can be used to gain additional insight into the psychological nature of musical structure by varying the type and composition of noncases. If a particular facet of musical structure is salient to the listener, one would expect that a noncase that violates that aspect of musical structure would be particularly noticeable to listeners. By observing which structural violations have the greatest impact on listeners' recogniton performance, one can infer which aspects of musical structure are most salient. In music, certain features have traditionally been considered to be more important than others. In baroque chorales, for example, the outer voices are supposed to be more important than the inner voices. Using noncases that violate the structure of either the inner or outer voices of the acquired idea would provide a behavioral test of this assertion. One may also ask if the salience of certain aspects of structure changes with the level of musical expertise of the listener. Experiments of this type would provide further insight into both the nature of musical structure and the nature of the abstraction of ideas.

\section{REFERENCE NOTES}

1. Pratt, R., Rushford, K., \& Jarrett, J. Variation of the Bransford and Franks effect using music. Unpublished manuscript, University of North Carolina at Oreensboro, 1973.

2. Chew, S. L., Blount, J., \& Waters, J. M. The abstraction of musical structure. Paper presented at the meeting of the American Psychological Association, Los Angeles, August 1981.

3. Serafine, M. L. The development of cognition in music. Paper presented at the joint meeting of the Society for Music Theory, the American Musicological Society, and the College Music Society, Denver, November 1980.

\section{REFERENCES}

Benjamin, W. E. Varieties of musical cognition: A music theorist's view. Journal of the Acoustical Society of America, 1981, 69, Supplement 1, S102.

Bransrord, J. D., \& Franks, J. J. The abstraction of linguistic ideas. Cognitive Psychology, 1971, 2, 331-350.

DEUTBCH, D. The processing of structured and unstructured tonal sequences. Perception \& Psychophysics, 1980, 28, 381-389.

Dowhing, W. J. Dichotic recognition of musical canons: Effects of leading ear and time lag between ears. Perception \& Psychophysics, 1978, 23, 321-325. (a)

Dowling, W. J. Scale and contour: Two components of a theory of memory for melodies. Psychological Review, 1978, 85, 341354. (b)

FLAGG, P. W. Semantic integration in sentence memory? Journal of Verbal Learning and Verbal Behavior, 1976, 15, 491-504.

Franks, J. J., \& Bransford, J. D. Abstraction of visual patterns. Journal of Experimental Psychology, 1971, 90, 65-74.

Jones, M. R. Auditory patterns: Studies in the perception of structure. In E. C. Carterette \& M. P. Friedman (Eds.), Handbook of perception (Vol. 8). New York: Academic Press, 1978. 
MEYER, L. B. Emotion and meaning in music. Chicago: University of Chicago Press, 1956.

MEYER, L. B. Explaining music: Essays and explorations. Berkeley: University of California Press, 1973.

Osgood, C. E., Sucr, G., \& Tannenbaum, P. H. The measurement of meaning. Urbana: University of Illinois Press, 1957.

Pick, A. D. Listening to melodies: Perceiving events. In A. D. Pick (Ed.), Perception and its development: A tribute to Eleanor $J$. Gibson. Hillsdale, N.J: Erlbaum, 1979.

Piston, W. Harmony (3rd ed.). New York: Norton, 1962.

Restle, F. Theory of serial pattern learning: Structural trees. Psychological Review, 1970, 77, 481-495.

\section{NOTES}

1. Experiment 2 was presented at the Midwestern Psychological Association, St. Louis, May 1980, under the title "Expert Knowledge and the Abstraction of Musical Ideas."

2. The authors wish to thank Robin Pratt for providing a tape of the Pratt et al. (Note 1) stimulus materials for use in this experiment.

(Received for publication December 14, 1981; revision accepted April 28, 1982.) 